\title{
GLAD!
}

Revue sur le langage, le genre, les sexualités

$02 \mid 2017$

Varia

\section{Dire le genre dans la presse magazine féminine et masculine}

Gender and Discourse in French Women's and Men's Lifestyle Magazines

\section{Aurélie Olivesi}

\section{(2) OpenEdition}

1 Journals

Édition électronique

URL : http://journals.openedition.org/glad/568

DOI : $10.4000 /$ glad.568

ISSN : 2551-0819

Éditeur

Association GSL

\section{Référence électronique}

Aurélie Olivesi, « Dire le genre dans la presse magazine féminine et masculine », GLAD! [En ligne], 02 | 2017, mis en ligne le 01 juin 2017, consulté le 21 janvier 2021. URL : http://journals.openedition.org/ glad/568; DOI : https://doi.org/10.4000/glad.568

Ce document a été généré automatiquement le 21 janvier 2021.

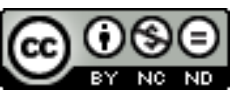

La revue GLAD! est mise à disposition selon les termes de la Licence Creative Commons Attribution Pas d'Utilisation Commerciale - Pas de Modification 4.0 International. 


\title{
Dire le genre dans la presse magazine féminine et masculine
}

\author{
Gender and Discourse in French Women's and Men's Lifestyle Magazines
}

\section{Aurélie Olivesi}

«Et si les Spice Girls avaient tout compris avant
tout le monde? Et si les filles étaient l'avenir de
l'homme? Et si les girls bandaient aussi?
Marion Ruggieri «Coups de girls », Elle, 4 janvier

2013

«Ce mois-ci, GQ entend vous démontrer qu'on peut être un homme apaisé et heureux dans un monde post-féministe. Tout comme on peut être un gentleman provocateur en étant le fils d'un comique de gauche." Emmanuel Poncet, « Regarde les hommes changer ", GQ, juin 2013

1 La comparaison de ces deux accroches d'éditoriaux, l'un écrit par une femme dans un magazine féminin et l'autre écrit par un homme dans un magazine masculin, publiés dans un intervalle de temps relativement bref sur un sujet similaire (les changements dans les rapports sociaux de sexe, appréhendés respectivement du point de vue féminin et masculin en fonction de leur lectorat) et s'adressant tous deux à un lectorat au genre défini, met en lumière un certain nombre de différences. Celles-ci - longueur des phrases, anglicismes, désignation des hommes et des femmes, références culturelles, questions ouvertes au style direct ou évocation d'un paradoxe - ont trait non seulement aux énoncés de ces éditoriaux (pris ici dans le sens de texte produit), mais également à leur énonciation, que l'on prendra dans la définition du « surgissement du sujet dans l'énoncé, [...] la relation que le locuteur entretient par le texte avec l'interlocuteur, ou [...] l'attitude du sujet parlant à l'égard de son énoncé » (Dubois 1969 : 100). Si l'analyse du discours médiatique portant sur le genre s'est largement intéressée aux énoncés (Coulomb-Gully \& Rennes 2010 : 178), la comparaison de ces 
deux extraits amène toutefois à s'interroger plus avant sur les modalités de la construction du genre à l'œuvre dans le discours médiatique: si le discours de ces magazines genrés diffère aussi bien sur le plan des énoncés du discours que sur le plan de leur énonciation, peut-on identifier un « discours de genre » dans les médias ? Nous entendons par « discours de genre » un discours qui se caractérise non seulement par la mise en œuvre de représentations de genre dans son énoncé, mais également par une performativité de genre inscrite au cœur même de son énonciation. L'enjeu est dès lors d'observer la dimension proprement discursive de la construction du genre dans les médias.

\section{Observer un « discours de genre » dans les médias}

2 Étudier la construction du genre dans le discours médiatique présente deux séries d'implications, ayant trait, d'une part, aux rapports entre construction du genre et construction du discours, et d'autre part - et réciproquement -, au rôle de ce discours médiatique dans la construction sociale du genre. En premier lieu, si Robin Lakoff a observé dès 1975 dans quelle mesure les hiérarchies de genre structuraient l'énonciation (Lakoff 2004 [1975]), c'est avec l'emprunt par Judith Butler (Butler 2005) du concept de "performativité " à la linguistique pragmatique que le lien entre les concepts clés de la construction du discours et ceux de la construction du genre a été mis en évidence ${ }^{1}$. Le concept de performativité semble dès lors central pour appréhender la construction du genre dans le discours (Benwell 2003). Cependant, les linguistes ayant amorcé ce lien ont souligné que les théories de Judith Butler demeuraient relativement décontextualisées et manquaient d'une analyse de textes existants $^{2}$ (Speer \& Potter 2002 ; Benwell 2003). En outre, l'idée de Judith Butler que « le genre n'est pas une entité stable et pré-discursive, inhérente aux individus, mais [qu'il] est construit, mobilisé et négocié dans l'énonciation du discours, que le genre n'est pas quelque chose que nous avons mais que nous "performons" " (Benwell 2003 : 151-152) remet largement en cause l'idée d'une dichotomie homme-femme pré-construite qu'il s'agirait ensuite d'observer dans un discours genré. Les pratiques discursives ne sont pas assignables a priori à une stratégie ou à une performance de genre ; le « discours de genre » est au contraire une construction du discours et du genre, et de l'un par l'autre réciproquement.

3 Ces premières observations amènent à s'interroger sur la seconde implication de l'étude de la construction du discours médiatique de genre : le rôle des médias dans la construction des rôles sociaux - et du genre en particulier - n'est pas neutre. Le discours médiatique véhicule des représentations de genre, mais il les construit également, comme le rappelle Teresa de Lauretis, pour qui les médias constituent une "technologie de genre " qui construisent le genre "en tant que représentation et autoreprésentation", autant qu'ils le représentent. Selon elle, il n'y a pas une représentation du genre à déconstruire, "la représentation du genre est sa construction » : elle « est construite par une technologie donnée, et, [elle est] d'autre part, [...] absorbée subjectivement par chaque individu à qui s'adresse cette technologie » (De Lauretis $2007:$ 41-65). Ainsi, les « technologies de genre variées [...] et [les] discours institutionnels [...] ont le pouvoir de contrôler le champ des significations sociales et donc de produire, promouvoir et "implanter" des représentations du genre " (ibid., p. 75). Si donc le discours médiatique produit le genre autant qu'il le représente, 
la dimension énonciative du discours médiatique peut se trouver autant structurante pour la construction du genre que les représentations véhiculées par le discours médiatique.

\section{Les éditoriaux des magazines genrés en 2013}

4 Afin d'observer un discours médiatique où le genre est énoncé de manière explicite, nous avons choisi comme corpus d'étude les éditoriaux de la presse magazine genrée (masculine et féminine), même si les magazines féminins sont beaucoup plus diffusés en France que les magazines masculins, type journalistique en constant renouvellement, et à la présence discontinue (Bardelot 2001 : 164). Le choix de ce type de presse a pour but de pallier une première difficulté, liée à ce que Françoise Héritier désigne comme «la valence différentielle des sexes » (Héritier 1996), qui se traduit, sur le plan linguistique, par la dichotomie entre caractère marqué du genre féminin et non marqué du genre masculin (Scott 1998:22). Cette valence différentielle est particulièrement visible dans le discours de la presse, dont l'universalisme proclamé se révèle en réalité fortement masculin : alors que le discours généraliste des médias se veut neutre et universel, il est en réalité écrit et lu en majorité par des hommes (Debras 2003 : 180). Paul McIlvenny a par ailleurs noté que la compréhension de la manière dont la masculinité "hégémonique » et les autres se construisaient dans le discours était encore à étudier (McIlvenny $2002: 11)^{3}$. Si les médias se présentent globalement comme destinés à un public mixte, la presse magazine s'adresse au contraire explicitement à des publics-cibles qui sont, pour certains titres, définis par leur identité de genre (masculine/féminine, hétérosexuelle ou LGBT). Dans la mesure où la presse magazine a pour particularité de ne jamais avoir distingué le lecteur du consommateur (Charon $2001: 66$ ), la définition du lecteur et la construction de son identité est très importante, car celui-ci est valorisé en tant que cible commerciale (Benwell $2004: 17$ ).

5 Nous avons souhaité neutraliser une deuxième spécificité du discours médiatique, sa polyphonie, en prenant en compte le discours dont nous avons par ailleurs montré qu'il était le plus « inclus » dans la presse écrite : celui des éditoriaux (Olivesi 2012 : 155-156). Le rôle de l'éditorial dans la presse d'information générale est traditionnellement d'apporter un point de vue à la fois commentarisant et surplombant sur l'actualité traitée dans le titre de presse $e^{4}$. Le discours des éditoriaux présente plusieurs spécificités : il est écrit par un-e seul-e journaliste, occupant une place élevée dans la hiérarchie de sa rédaction et qui s'exprime au nom de la rédaction tout entière; il ne comporte pas non plus de discours rapporté. Les éditorialistes occupent des positions élevées au sein de la hiérarchie des rédactions et appartiennent à ce que Rémy Rieffel désigne comme « l'élite des journalistes » (Rieffel 1985). Ils et elles bénéficient à ce titre de tribunes dans les autres médias. Ainsi, les éditoriaux des magazines peuvent occuper une place dans la constitution du débat public sur la définition de la masculinité, de la féminité et de leurs rapports. Il s'agit donc, même s'il est difficile de savoir si l'éditorial des magazines est beaucoup ou peu lu, d'un discours qui ancre les représentations dans l'espace public médiatique. Serge Guérin, qui a analysé les éditoriaux du magazine Notre Temps, a soulevé une autre spécificité des éditoriaux dans la presse magazine: leur dimension programmatique sur le contenu et le positionnement du magazine. Selon lui en effet, «si la couverture exerce une fonction "vitrine" du journal, l'éditorial structure et définit le positionnement du support » (Guérin 1999: 11). Or, dans la mesure où les magazines que nous prenons en compte s'adressent explicitement à un public genré (à 
la différence des magazines politiques, économiques ou sportifs, par exemple), on peut considérer que l'éditorial est susceptible de définir l'orientation du journal et son positionnement à l'égard du genre.

6 Nous avons choisi pour année de référence l'année 2013, car elle a vu l'apparition d'un questionnement sur la masculinité dans la presse : plusieurs dossiers sur des supposés " nouveaux hommes » au printemps ( $G Q$, Causette, Books ${ }^{5}$ ), et l'apparition (ou la réédition) de deux nouveaux magazines masculins : Lui, et Elle Man à l'automne. 2013 a également vu éclore plusieurs débats liés aux questions de genre : la question de l'ouverture du mariage aux couples homosexuels (loi votée le 17 mai 2013), le débat sur la mise en place des «ABCD de l'égalité » dans les écoles ${ }^{6}$, le manifeste des « 343 salauds » publié dans le magazine Causeur au mois de novembre ${ }^{7}$.

7 Le corpus que nous avons choisi est le suivant : les éditoriaux de GQ, car il s'agit selon l'Alliance pour les Chiffres de la Presse et des Médias du magazine masculin le plus lu ${ }^{8}$, et ceux de Lui et de Elle Man. L'analyse du discours de magazines féminins (nécessairement plus nombreux et plus diffusés) servira de contre-point à cette définition : Elle (d'où est issu Elle Man) et Marie-Claire (mensuel, à l'instar des magazines masculins). Nous avons choisi de prendre des magazines féminins « haut de gamme » (Darras 2004: 273) pour faire le pendant de ces journaux, dans la mesure où les magazines masculins que nous avons observés s'adressent à des hommes à fort pouvoir d'achat (Bardelot 2001). Par ailleurs, les magazines moins "haut de gamme", pour reprendre l'expression d'Éric Darras, n'ont soit pas d'éditorial, soit un éditorial très court. Nous avons également adjoint à ce corpus les éditoriaux du magazine féministe Causette (dans ce magazine, l'éditorial est anonyme, mais la signataire, Causette, est explicitement identifiée comme une femme), avec comme corpus annexe au corpus masculin l'éditorial du supplément Causette Monsieur paru en août. Nous n'avons pas inclus Têtu, ni aucun magazine dédié au public LGBT, car si les questions liées au genre sont au cœur des problématiques de ces magazines, l'année 2013 était celle du vote sur le mariage pour tous, et cette thématique a occupé la majeure partie des éditoriaux.

\section{Énoncé et énonciation d'un « discours de genre » : ancrage collectif et recours à l'ironie}

8 L'hypothèse que l'on peut formuler est double. En premier lieu, il s'agit de montrer que l'expression du genre dans le discours journalistique genré n'est pas un décalque d'une dichotomie homme/femme figée. Elle est en revanche révélatrice des mécanismes discursifs à l'œuvre dans la performativité de genre et la construction du genre. Le second volet de notre hypothèse s'attache à montrer que la construction discursive du genre se trouve moins dans une différence de thématiques que sur le plan de l'énonciation, et que le point central de cette construction est l'usage de l'ironie à l'égard de soi-même ou de son propre discours. Nous entendons montrer que la construction du genre se traduit dans le discours par le rapport du locuteur à sa propre énonciation : ironique dans les magazines masculins et féministes, militante - mais implicitement - dans les magazines féminins "classiques ». Le rôle joué par l'ironie dans la construction de la figure de l'énonciateur/trice, en tant qu'il est constitutif d'une posture surplombante dans une connivence avec son lectorat, est particulièrement structurant dans la construction d'un « discours de genre ». C'est dès lors sur le plan énonciatif que se joue très largement la construction du genre dans le 
discours médiatique et que peut s'observer une évolution dans les expressions discursives du genre.

Nous examinons dans un premier temps les thématiques abordées, pour montrer qu'elles sont légèrement en décalage avec ce que Éric Darras notait du contenu général des articles de la presse féminine, à savoir un recentrement sur la sphère privée (Darras 2004 : 275). Nous verrons ainsi que la divergence des thématiques entre magazines féminins et masculins renferme une convergence de perspectives, pour s'accorder sur une profonde évolution des rôles de genre. On observera dans un deuxième temps les termes qui dénotent les rapports sociaux de sexe (féminisme, machisme, genre). En effet, la presse féminine décrit la féminité à l'aune d'un hic et nunc mondialisé, alors que la presse masculine décrit sa masculinité programmatique dans un héritage, lui-même extrêmement français. Enfin, dans un troisième temps, nous analyserons l'énonciation de ces éditoriaux pour observer une dichotomie dans la construction du genre qui ne s'opère pas en fonction du genre du lectorat, mais en fonction du recours à l'ironie dans le discours de l'éditorial. Alors que l'ironie du locuteur à l'égard de sa personne et de son discours est omniprésente dans les magazines masculins et féministe, elle est quasiment absente des magazines féminins traditionnels. Dès lors, le positionnement énonciatif (ironique ou non) se révèle particulièrement structurant dans la construction d'un « discours de genre ».

\section{Les thématiques mises en œuvre : de la distinction « dedans » / " dehors » à la convergence des enjeux}

10 Pour observer une différence dans la contribution au débat public sur la définition du genre au sein des éditoriaux, nous nous sommes dans un premier temps intéressée aux thématiques développées dans ces articles, à partir du titre et du contenu des éditoriaux.

11 L'analyse des titres des éditoriaux montre que les thématiques recoupent largement l'opposition entre, comme le dit Éric Darras, le « dehors » et le « dedans ». Selon lui, « la division sexuelle des pratiques de lecture de la presse magazine traduit et travaille à reproduire la division sexuelle du travail entre l'intérieur et l'extérieur " (Darras 2004 : 273-274). Ainsi, dans Marie-Claire, qui a commencé à publier un éditorial en mars 2013, outre deux sujets sur la mode, on trouve, dans l'ordre chronologique,

- la procréation médicalement assistée

- le corps des femmes

- la contraception

- l'accouchement sans péridurale

- le bonheur

- les femmes et le travail

- l'éducation des garçons

- la négociation du salaire

Dans le même temps, Causette, magazine féministe, parle dans ses éditoriaux de la publicisation du corps des femmes, des problèmes de garde en crèche, etc. Les thématiques traitées dans les éditoriaux des magazines féminins parlent effectivement de problématiques liées à la vie privée. Du côté des magazines masculins, les éditoriaux semblent largement centrés sur l'identité du magazine : les éditoriaux du magazine $G Q$ 
parlent du magazine $G Q$ lui-même et des palmarès qu'il met en œuvre, ou bien de la réussite et du changement des hommes. De même, Lui parle de Lui, Elle Man parle de Elle Man. Si ces journaux parlent autant d'eux-mêmes, on peut penser que c'est parce que la place d'un journal masculin est encore relativement difficile à définir. Ainsi, l'éditorial de Lui fait une large place à la personne de son rédacteur, Frédéric Beigbeder, écrivain mondain et ancien publicitaire. Cependant, les hommes représentés ne sont pas envisagés en lien avec leur espace intime ou domestique, mais ancrés dans l'espace social.

Si la dichotomie extérieur/intérieur semble présente, lorsque l'on observe le traitement de ces sujets, on perçoit cependant moins une opposition de thématiques figées que des perspectives plus larges, envisagées de surcroît de manière dynamique. En effet, les magazines féminins partent de problématiques spécifiques pour aboutir à une perspective globale sur la place des femmes. Afin d'observer les implications de ces représentations genrées, nous avons repéré à l'intérieur du discours de chaque éditorial les termes qui dénotaient le féminin et le masculin ("femme", "homme", «mâle ", jusqu'au pronom «nous »). Dans Marie-Claire, les sujets traités dans l'éditorial sont inscrits par Christine Leiritz, directrice de la rédaction, dans une perspective plus large, qui est une perspective politique et mondiale :

(1) «De Kaboul, Paris, Bruxelles ou Bogota, elles sont toutes là - femmes du monde, femmes en lutte, femmes d'à côté -, dans ce nouveau Marie Claire. Où, sinon? Qui, pour faire le lien entre elles et vous, vous et nous ?» (mars)

(2) « On naît toutes quelque part. En Inde, en Afghanistan, aux Pays-Bas, en Egypte, aux Etats-Unis, en Ukraine, en France. On naît femme quelque part, on n'a pas choisi où, on n'a pas choisi son sexe, mais on va le payer. Ici contrainte de marcher le visage caché, là excisée à 6 ans, violée sur un campus, tabassée, mariée à 11 ans, c'est la même histoire » (mai)

Dans ces deux extraits, la locutrice combine les trois mêmes éléments : une énonciation à la première personne du pluriel, une juxtaposition de situations aussi variées sur le plan thématique que géographique et une perspective généralisante et englobante. Le magazine a pour perspective de montrer que les problématiques relatives à la condition féminine sont toutes liées. L'éditorial sur la pilule est l'occasion de revenir aux problématiques de la libre sexualité, celui sur le salaire d'évoquer les blocages sociaux dans le monde professionnel: "Vous faites probablement partie des $66 \%$ de femmes qui s'avèrent incapables de réclamer une augmentation, c'est du moins le chiffre avancé dans une étude menée dans dix-sept pays européens » (Marie-Claire, décembre). La perspective est identique dans Marie-Claire, Elle et Causette. Ainsi, la manière dont les magazines parlent des sujets dits "féminins" est une manière d'ancrer des problématiques féminines dans les discussions politiques, comme l'avait noté Sylvie Debras (Debras 1999: 121). Causette note ainsi que «[1]e problème pour nous, c'est qu'entre l'avortement, le débat sur la PMA, la contraception, nos mille sécheresses et autres prétendus besoins de toilette "intime", l'intérieur du corps féminin est en permanence investigué par l'œil public. Et que l'inégalité est aussi là " (Causette, janvier). On retrouve dans cet extrait le "nous» englobant, la juxtaposition des situations et la généralisation de la perspective à toutes les femmes.

Si les magazines féminins élargissent leur problématique, les magazines masculins partent de leur posture éditoriale novatrice et spécifique pour définir de la même manière une nouvelle place des hommes. On trouve ainsi dans $G Q$ un éditorial intitulé 
"Regarde les hommes changer» (juin) qui proclame que "les hommes ne sont réductibles à aucun éternel masculin qu'il faudrait perpétuer depuis la nuit des temps, mais bien une infinité de traits physiques et psychologiques, hérités biologiquement ou socialement construits, avec lesquels on peut vivre très bien, merci, et jouer surtout ». La même idée se retrouve dans Elle Man : «C'est l'histoire d'un mec. Alors, qui est-il ? Il n'en sait rien et il s'en fout ", avant d'esquisser une définition : « Un garçon qui assume son style, qui dépasse son genre, qui va bien et qui ne s'interdit rien » (octobre). De la même manière, Causette Monsieur, qui reprend pour titre de son éditorial le slogan publicitaire de l'entreprise de rasoirs Gillette («Pour nous, les hommes»), rejette les représentations non questionnées de la masculinité : «Lorsque tu prononces ces mots dans ta pub, mec, j'ai l'impression de devoir me coltiner un vieux sac à dos bien lourd de clichés» (août). Dans le cas des magazines féminins, comme masculins, la perspective est résolument constructiviste.

La figure repoussoir est ainsi celle de "tous les vieux mâles dominants » également appelés «vieux mâles nostalgiques de la loi du Père » $(G Q$, juin), présente également dans Causette: "Mon oncle, il fait partie de ces hommes qui ont décidé de changer de Régime. D'abandonner le vieux modèle du mâle à l'ancienne et ses privilèges de dominant " (mars). Cette figure est définie en référence à "un éternel masculin supposé [...] des idées périmées, des mœurs poussiéreuses, de vieilles lunes et, pour tout dire, d'hommes dépassés " (Causette Monsieur, août). Il est à noter que ces journaux précisent que leur approche constructiviste se distingue d'un discours conservateur extrêmement virulent (Dupuis-Déri 2012:129), comme le rappellent à la fois Causette et GQ. Causette évoque le discours assimilant le changement dans les rôles de genre à une castration « [q]ui souffle [à son oncle qu']il ne sera même plus un homme ou, alors, un homme à la manque, une espèce amputée. Castrée!» (Causette, février), évocation également présente dans $G Q$ : "Cela fait déjà plusieurs années que les médias nous rebattent les oreilles avec une prétendue crise de la masculinité [...] "Féminisés", voire "émasculés", les hommes se voient affublés d'improbables qualificatifs - métrosexuels, übersexuels, rétrosexuels... [...] il n'aura pas échappé au lecteur de $G Q$ que l'apocalypse masculine, comme celle des Mayas, est un curieux leitmotiv de notre temps " (juin). Quant aux locuteurs cités, ils sont les mêmes, et dans le même ordre : en premier lieu "des "masculinistes", spécialisés dans le détournement médiatique du patrimoine urbain, qui dénoncent ces "bonnes femmes qui nous gouvernent, [et qui] se foutent toujours de la gueule des papas" » dans Causette (mars), qui sont évoqués également dans $G Q$ « obligés de grimper sur des grues pour se faire entendre... » (juin), et d'autre part « des sociologues autoproclamés qui prédisent rien de moins que la Fin des hommes [et] jouent aux Nostradamus de comptoir sur la thématique de la disparition de l'homme viril ; du père; de l'homme, le vrai » (Causette, mars) que l'on retrouve dans $G Q$ : «De l'essayiste Éric Zemmour qui se lamentait dès 2006 de la "dévirilisation" des hommes, comme s'il s'agissait d'une dévaluation du franc sous Raymond Barre, à la journaliste Hanna Rosin qui prédit carrément cette année dans un ouvrage surmédiatisé The End of Men (éd. Autrement)» (juin). Si ces deux médias citent les mêmes discours opposés, c'est que le "discours de genre " mis en place dans ces magazines, se construit donc comme un discours positif et novateur, en opposition à une multitude d'autres discours, tous négatifs et nostalgiques. Les places de genre traditionnelles sont montrées comme dépassées, puisque la posture considérée comme "traditionnelle» se voit qualifiée d'un terme issu des confrontations intellectuelles suscitées par le féminisme : le «masculinisme ${ }^{9}$ ». Ainsi, l'éditorial du premier numéro 
de Lui, qui donne des hommes et des femmes des définitions figées, affirme son caractère rétrograde ("Cet été, à Biarritz, j’ai vu les derniers hommes sur la plage. [...] Lui est ma dernière tentative pour rester vaguement masculin. [...] Le retour de Lui, c'est le plaisir d'un dernier tour de piste, c'est un baroud d'honneur en souvenir de ce dinosaure nommé le Mec »), que Frédéric Beigbeder qualifiera avec joie de "vintage » au numéro 2, mais qu'il inscrira dans cette même tension dans son éditorial numéro 3, au mois de décembre: "Tel Monsieur Jourdain, je viens d'apprendre que j'étais masculiniste sans le savoir ». Et de reprendre les mêmes exemples, mais avec un point de vue inverse :

(3) De même que les féministes défendent les droits des femmes, est masculiniste toute personne qui milite pour les droits des hommes. Par exemple, le droit pour un père de voir son enfant en cas de divorce sans être obligé de monter sur une grue, ou le droit pour un homme qui s'est fait faire un enfant dans le dos de ne pas se faire racketter pendant dix-huit ans par une femme qu'il n'a vue qu'un soir dans sa vie.

La posture de Lui, qui se revendique rétrograde, s'oppose donc à celle des autres magazines qui se positionnent dans une redéfinition des places de genre.

Cette redéfinition se retrouve également dans les magazines féminins non explicitement féministes, comme Marie-Claire: «il est temps d'appliquer à ceux que nous avons enfantés ce qu'on aimerait qu'on nous applique à nous-mêmes : le droit d'être ce qu'on est, qu'on soit un homme ou une femme " (novembre), et dans Elle avec un retournement des caractéristiques de genre " "Ce qui me plait chez une femme, c'est sa part de virilité", la phrase est de Catherine Deneuve, qui n'en manque pas » (Elle, 20 septembre). Cette redéfinition passe dans les magazines féminins par une réappropriation des caractéristiques traditionnellement associées à la masculinité, que ces caractéristiques soient montrées comme construites, comme dans Marie-Claire ( « nous retiendrons l'audace, le goût d'entreprendre, la combativité, la confiance en soi, toutes qualités réputées masculines ", Marie-Claire, septembre) ou naturalisées, comme dans Elle, qui évoque « l'appropriation d'éléments masculins à un nouvel usage féminin. Qu'y a-t-il de plus viril et caréné que des bottes de moto, qu'y a-t-il de moins sexué qu'une paire de baskets ? Qu'y a-t-il de plus "gentleman" que tous ces derbys [...] ? Qu'y a-t-il surtout de plus rapide, dynamique et conquérant que toute cette platitude ?» (Elle, 25 octobre). On peut parler de naturalisation dans la mesure où les trois adjectifs "rapide, dynamique et conquérant" font écho à deux caractéristiques dénotant explicitement la masculinité : "viril " et " "gentleman" ». L'appropriation pour être aboutie doit, toujours selon Elle, être réciproque : face à des " garçons [qui] rament, ne comprennent plus, se plantent [...], une génération de boys hyper mutiques, hyper paumés, hyper touchants. La solution, ce serait évidemment de leur apprendre à mettre des mots sur leurs maux. Les garçons, il est grand temps de vous mettre à table "(Elle, 29 novembre). Quels que soient leur lectorat et leur ligne éditoriale, les différents magazines adoptent une posture similaire dans le débat public sur des rapports de genre en évolution. On y retrouve la «contradiction dynamique » entre «invention» et "standardisation» propre aux industries culturelles telle que l'a montrée Edgar Morin (Morin 1962: 43) : il s'agit à la fois de plaire au plus grand nombre, ce qui induit un certain conformisme, tout en se renouvelant, à la fois pour se démarquer de la concurrence et pour accompagner les évolutions sociales (Macé 2001 : 7). Ainsi, les énoncés des éditoriaux dans les magazines féminins et masculins ne 
recoupent pas une dichotomie hommes-femmes figée, mais font montre au contraire d'une convergence dans l'effort de rendre compte de rapports de genre en pleine reconfiguration. Dans cette perspective, il faut se rappeler que notre corpus est un corpus « haut de gamme » et ne pas oublier que la variable de genre doit être modérée par la variable sociale: dans la mesure où ces magazines s'adressent tous à des catégories socioprofessionnelles supérieures, les thématiques tendent à se recouper. Cependant, les définitions du genre masculin et féminin se différencient dans leur ancrage. Les réseaux de significations dans lesquelles ces représentations s'inscrivent (Jodelet $2003: 56$ ) demeurent distincts.

\section{Féminité de lutte vs masculinité héritée}

Les éditoriaux placent le discours de genre dans deux dynamiques différenciées. La féminité est présentée comme une féminité de lutte, alors que la masculinité est montrée comme un héritage (Connell $2005: 82$ ). Si le féminisme est présent dans les magazines s'adressant aux femmes, il demeure implicite dans Marie-Claire, contradictoire dans Elle, alors qu'il est affirmé et explicite dans Causette.

\section{Le féminisme : un combat dépassé ?}

20 Si l'approche constructiviste de l'identité de genre est bien héritée d'une approche féministe, il ne faut pas perdre de vue que le féminisme en lui-même est présent implicitement dans la plupart des éditoriaux des magazines féminins - sans être assumé explicitement pour autant. Alexie Geers note ainsi que «alors même que la référence aux questionnements féministes est permanente [dans Marie-Claire], la rédaction ne se revendique jamais du mouvement ni ne rend compte de ses actions politiques» (Geers 2016:253). Dans Marie-Claire se trouve ainsi un éditorial intitulé "Vibrer à l'unisson de vos combats " (mars) et un autre "Liberté, égalité, féminité " (mai). La pilule est assimilée à « la maîtrise de nos choix personnels et professionnels. Le prix de notre liberté, faute de mieux » (juin), et le "retour à la nature », comme la justification de comportements sociaux défavorables aux femmes: «La nature, c'est net, veut reprendre ses droits, ou plutôt les nôtres. Elle réclame désormais de nombreux aménagements de la vie moderne [...] qui malheureusement tombent souvent sur les mêmes : les femmes. Notre fonction première, comme chacun sait, étant d'enfanter [...]. La nature, c'est comme Dieu. On a vite fait, au gré de ses interprètes, de lui faire dire ce qu'on veut " (juillet). De la même manière, une approche féministe constructiviste est énoncée dans l'éditorial du mois de novembre, où l'on retrouve les résultats de Catherine Vidal (Vidal \& Benoit-Browaeys 2005), sans que cette dernière ne soit citée explicitement. L'éditorialiste dénonce en effet :

(4) une cruelle méconnaissance du cerveau humain, qui ne prédestine aucunement les filles à réussir à l'école, et les garçons, à s'y montrer des cancres, car il se trouve que c'est le même, quel que soit le sexe [...]. [et qui] corrobore [...] la théorie selon laquelle c'est précisément les a priori sur la nature supposée des deux sexes (garçons actifs, en mouvement, peu enclins à la concentration, vs filles passives, statiques, attentives à leur ouvrage), qui génère, via l'éducation, ces fameuses différences de comportement à l'école. (novembre) 
21 L'identité féminine est présentée comme construite, et n'est jamais détachée des problématiques du féminisme. Cependant, le féminisme qui est affirmé dans ces éditoriaux demeure épidermique et réactif à l'actualité : les féministes évoquées dans Marie-Claire sont soit « une bande de vieilles féministes old school [, soit] quelques jeunes Femen dépoitraillées » (septembre). Dans un éditorial intitulé « Trop de féminismes ? ", Elle donne de son côté du féminisme des définitions divergentes, et parfois mêmes contradictoires :

(5) C'est à n'y plus rien comprendre. Un vrai méli-mélo, ces féministes ! [...] Le 8 mars approche à grands pas et avec cette Journée de la femme, [...] comme il fait bon se déclarer féministe ! [...] est-on autorisé à rêver mieux ? À un féminisme adulte et généreux, qui ne serait pas un masque mis sur d'indicibles et ancestrales pulsions : ni une haine des hommes et de leur désir sexuel ni une haine des femmes et de leur désir sexuel. [...] Un féminisme dupe de rien et surtout pas de lui-même. [...] Une prise de risque. La vie même. (1 er $^{\mathrm{r}}$ mars)

Si l'on retrouve une des critiques traditionnellement adressées au féminisme: le puritanisme (Bard 2012 : 237), le féminisme est présenté dans cet extrait comme vivace au passé et au futur, mais problématique au présent. Les magazines masculins relèguent eux aussi le féminisme au passé, qu'ils s'en réjouissent, comme GQ qui se place « dans un monde post-féministe » (juin), ou le déplorent, comme Lui, qui affirme que les femmes "ont gagné la guerre" (septembre). Causette constitue ainsi une exception notable, mais son propos est explicitement féministe : "J'ai écrit, il y a bien longtemps, cette petite phrase: "Le féminisme, c'est comme le ménage, si on ne s'y colle pas régulièrement, on finit par s'habituer à la crasse" " (Causette mars), où l'on remarque que le féminisme est présenté au singulier, au présent et avec une nécessaire itération.

On peut toutefois noter une différence entre les différents magazines lorsque la référence est faite aux figures qui incarnent le féminisme. On en trouve sept dans notre corpus : Clémentine Autain, Elisabeth Badinter, Les Chiennes de Garde, les Pussy Riot, les Femen, Simone de Beauvoir et Gisèle Halimi. Leur traitement diffère sensiblement en fonction du titre où elles sont citées. La figure (collective) la plus présente est celle des Femen, citées à la fois par Marie-Claire et Elle, pour leur action récente et concrète (dans Elle: évocation en janvier, citation du livre collectif Nudity is freedom en mars, mention de la jeune Tunisienne Amina Sbouï en juillet), pour ne pas dire anecdotique («Quelques jeunes Femen dépoitraillées» dans Marie-Claire en septembre). On retrouve le même traitement anecdotique de Simone de Beauvoir dans Elle, où elle est également citée pour une action concrète : le Manifeste des 343 salopes (8 novembre), alors que la même Simone de Beauvoir est citée dans Causette, comme une figure tutélaire : "Tout ce que je sais, c'est que Bachar en Simone de Beauvoir, j'ai un peu de mal à y croire " (mars). Se dessine une différence entre la citation de figures féministes soit comme exemples ponctuels, soit comme figures de référence, aux influences potentiellement plus larges, tout en demeurant implicites. C'est le cas de Gisèle Halimi dans GQ, puisqu'il est rapporté que Nicolas Bedos (lui-même non dénué d'héritage explicite car fils du comique Guy Bedos) enfant sautait sur ses genoux (juin). Cette présence de figures tutélaires qui n'appartiennent pas au temps présent, mais constituent un ancrage culturel, constitue une différence notable entre le discours des magazines masculins et celui des magazines féminins. Lorsque l'on observe les personnalités citées 
explicitement dans les éditoriaux des magazines masculins, on observe que, à la différence de Elle (3,057 références explicites par éditorial), de Marie-Claire $(0,8)$ ou de Causette $(0,91)$, où les références présentes sont pour majeure partie liées à l'actualité immédiate, les références sont dans $G Q(9,42)$ et $L u i(7)$ non seulement plus fréquentes, mais plus variées. Elle Man, avec 4,5 références en moyenne par éditorial - appartenant toutes à l'actualité culturelle, artistique et audiovisuelle - semble plus proche de son média de tutelle.

\section{L'effet de connivence des références culturelles} très hétéroclite de domaines culturels. On y trouve en premier lieu des références culturelles appartenant à des domaines que Bourdieu désignait comme légitimes (Bourdieu 1979), avec des citations au style direct dûment référencées, comme la philosophie (Nietzsche, $G Q$, avril ; Jean-Paul Sartre, Lui, décembre), la littérature (dans Lui : Don Quichotte, en octobre, Michel Houellebecq et Le Bourgeois Gentilhomme en décembre ; Céline et Oscar Wilde dans GQ, février et novembre) - non sans une certaine ironie : «"Gagnez les profondeurs : l'ironie n'y descend pas”, professait à tort le poète (Rilke, pour nos lecteurs normaliens) » $G Q$, août) -, ou la peinture. Lui énumère ainsi " Michel-Ange, Botticelli, Ingres, Boucher, Courbet [...] Magritte " dans son numéro 2 avant d'évoquer «les films underground de Jonas Mekas sur Andy Warhol ou le mouvement Fluxus » dans son numéro 3. Mais on trouve également la référence à des pratiques culturelles plus populaires: citations de films d'Yves Robert (GQ, Juillet, novembre), allusions à un titre de film de Jacques Audiard (GQ, juin), à Coluche (Elle Man, octobre), interview du judoka Teddy Riner (GQ, février)... Les références des magazines masculins juxtaposent des domaines légitimes à des domaines plus populaires, composant ce que Bernard Lahire nomme une "culture des individus " (Lahire $2004: 688$ ) dissonante, fondée à la fois sur des plaisirs exigeants et d'autres plus faciles d'accès - sans que ces médias semblent pour autant dresser une hiérarchie entre ces deux champs culturels ${ }^{10}$.

Les références culturelles sont beaucoup plus ténues dans la presse féminine. On repère dans Causette une allusion à Brassens («Un cerveau frigide dans une peau de vache »; avril), une à Delacroix (août) et une citation historique (Arnaud Amaury lors du siège de Béziers en 1208) : «Tuez-les tous!» (octobre). Quant au «Tu ne seras pas un con, mon garçon » de Marie-Claire (novembre), on y retrouve en filigrane Rudyard Kipling. Mais la référence culturelle n'est jamais explicitée plus avant. On trouve bien dans Causette un long récit de l'histoire des sœurs Mirabal dans la République dominicaine de Rafael Trujillo en novembre, mais le titre de l'éditorial «Connaissez-vous l'histoire des sœurs Mirabal ?» signale bien évidemment que cette histoire n'est pas connue.

La présence de la culture produit un effet de continuité entre figures tutélaires, « cover boys" (selon la terminologie de $G Q$ ) et lecteurs-consommateurs. De même, les « cover girls » de Lui (Léa Seydoux, Georgia May Jagger, Edita Vilkeviciute et Malgosia Bela) sont magnifiées non seulement physiquement, mais socialement (comme appartenant à une élite sociale, ou professionnelle dans le mannequinat), créant ainsi une impression de connivence entre le magazine et son lectorat. On ne trouve pas d'équivalent dans la presse féminine, où la couverture est distincte de l'éditorial. La culture est ainsi prise, de même que la référence aux « cover-boys ", dans une logique d'héritage, qui fait de 
ces hommes des figures tutélaires, ou du moins des "role models», des mentors, comme Fabrice Lucchini et Teddy Riner, « deux géants brillants dans leurs domaines respectifs (et même au-delà), deux hommes qui cumulent puissance physique et maturité intellectuelle, deux hommes qui "envoient" comme on dit » (GQ, février). En juillet, " l'immense Pierre Richard, dont la célèbre chaussure noire est assimilée à un autre modèle, un "agnellisme", en référence au dandy Giovanni Agnelli, le fondateur de Fiat, qui cultivait malicieusement les erreurs de style » est montré à la fois comme héritier et comme précurseur, puisque $G Q$ le voit « incarn[er] à la fois l'un des héritiers du gag subversif à la Chaplin et le parrain LOL de la nouvelle génération comique ». La constitution d'une sorte de communauté du magazine vers la personne mise en couverture via l'éditorial, d'une part, et de l'éditorial vers ses lecteurs, d'autre part, crée un effet valorisant de filiation, à la fois culturel et national.

\section{Les contradictions de l' « esprit français »}

Si les références culturelles appartenant à la culture "légitime » sont internationales, les références aux produits issus des industries culturelles appartiennent toutes à la culture française, produisant un effet de connivence et d'héritage. Pierre Richard est ainsi présenté comme incarnant «un certain art du burlesque à la française » (GQ, juillet). Cet ancrage français est explicité par $G Q$, évoquant les problématiques de son édition française, lancée en 2008 :

(6) En février 2008, les journaux français continuent de perdre des lecteurs, et la presse masculine souffre d'une image souvent désastreuse, «bling » et "beauf». Au mieux, une "presse de niche» à destination des dandys fumeurs de cigares, au pire une "presse de miches" à base d'abdos et de bimbos. Le lancement de $G Q$ vient démentir tous ces préjugés, alimentés par l'absence d'une culture française de l'entre soi masculin (à la différence des clubs anglais ou des fraternités américaines). (GQ, mars)

Le lancement d'une édition française d'un magazine international s'appuie ainsi sur les spécificités nationales à la fois de la presse et de l'expression de la masculinité. MarieClaire justifie son ancrage international de manière presque strictement inverse en mars : «La vie, un jardin [...] dont on détectera pour vous les tendances les plus signifiantes : c'est notre force de média international ». Le Français décrit par GQ est un Français mondialisé : « Ils ne considèrent pas [...] la France en prédateurs [...]. Pour eux, [...] la France et le reste du monde ne s'opposent pas. Ils se nourrissent mutuellement » (GQ, avril). C'est l'image de la culture des élites présentée par Anne-Catherine Wagner, qui rappelle que "l'ouverture sur l'étranger est un attribut distinctif des classes supérieures »: au "cosmopolitisme des vieilles familles» se superpose "l'internationalisation plus récente d'autres fractions des classes supérieures" (Wagner 2007: 17). Ainsi, la vision de la masculinité française proposée par ces magazines n'est pas distincte d'un ancrage social présenté comme bourgeois (à rapporter au fait qu'il s'agisse de magazines « haut de gamme»), alors que les médias anglo-saxons promeuvent un modèle dit du « New Lad » où la masculinité est associée à des valeurs issues des classes populaires (Young 2016). Ainsi, même quand la référence est faite à une série américaine, L'homme qui valait trois milliards ${ }^{11}$, c'est sa diffusion française qui est évoquée : «The Six Million Dollar Man en Vo, soit une énorme plus-value monétaire lors de la traduction française, à moins qu'il ne s'agisse d'anciens francs. [...] Beaucoup d'entre nous ont gardé dans l'oreille le "tut tut tut" émis par son œil 
bionique - "bioyonique" disait drôlatiquement la VF - quand il visait une cible " (GQ, août). Ainsi, de même que les références à la culture française structurent le portrait de Fabrice Lucchini, «ambassadeur de la culture à la française ", qui est dit capable « de citer Céline et Yves Calvi de "C dans l'air" " (GQ, février), les hommes mis en avant dans l'éditorial sont présentés comme ayant les mêmes pratiques culturelles que l'éditorialiste - qu'il attribue également à son lecteur, dans une filiation directe des uns aux autres.

L'esprit «français » est par ailleurs décrit comme caractérisé par ses contradictions, comme on le voit à propos de Fabrice Lucchini, « ambassadeur flamboyant de toutes les contradictions de l'esprit français (aisance et préciosité, goût du populaire et snobisme avéré, libertaire et réactionnaire...) » (GQ, février) ou de Vincent Cassel, qui « sembl[e] incarner mieux que personne l'"homme français GQ", dans sa complexité, sa plasticité, ses aspérités. Élégant et brut. Physique et cérébral. Instinctif et spirituel. Bourge et voyou » (GQ, mai). Le magazine Lui construit dans son premier numéro également une figure d'homme présenté comme hédoniste et contradictoire ( Ce mammifère viril et romantique, obsédé par les femmes et ami des gays, insupportable et sexy, luxueux et paysan, festif et littéraire ») et désigné comme un "Français »: «On aurait aussi pu dire "Français", si cela ne risquait pas de créer un sordide malentendu avec des personnes nationalistes » (Lui, septembre). Ce rejet d'une identité française repliée sur elle-même se retrouve dans la figure-repoussoir du «franchouillard». Ainsi, Vincent Cassel, dans un éditorial : "Une autre idée de la "win" » (GQ, avril) (où l'on retrouve le lointain écho d'" une certaine idée de la France » énoncée par de Gaulle en incipit des Mémoires de guerre), est présenté comme « désamorçant à lui seul toutes les polémiques franchouillardes " (GQ, mai). La masculinité programmatique est parfois progressiste, parfois nostalgique, mais elle a les mêmes composantes culturelles françaises des classes supérieures, combinées à une dimension oxymorique: "Nos critères? Objectivité factuelle et subjectivité assumée. Souci du style et air du temps. Bon sens et arbitraire " (GQ, décembre), qui vise à traduire une certaine complexité, mais également à montrer la distance à soi-même impliquée dans ces définitions. Or, ce rapport ironique à sa propre énonciation, s'il est très présent dans les magazines masculins, est globalement absent des éditoriaux des magazines féminins de notre corpus. On ne le retrouve que dans le contexte de propos explicitement énoncés comme féministes. La question du recours à l'humour et à l'ironie semble donc être au centre d'une recomposition du discours de genre.

\section{Le rôle central de l'énonciation ironique}

L'énonciation ironique est au cœur de la construction du genre dans le discours. Nous prendrons ici le terme ironie dans son sens minimal «moderne et courant» de «sorte d'antiphrase qui consiste à faire entendre ce que l'on veut dire en disant précisément le contraire, avec une intention de moquerie " (Lalande 2010 [1926] : 544-545). Le sens "médiéval » relevé par Lalande d'" acte de se déprécier par un mensonge [...] pour se faire valoir par contraste» et qu'il dit être "tombé en désuétude»(ibid.) semble toutefois également opératoire pour analyser les textes composant notre corpus. En effet, le discours des magazines masculins et féministe donne des locuteurs, des personnalités de référence et du lectorat des représentations paradoxales, oxymoriques, humoristiques, qui ne semblent pas toujours valorisantes sur le plan des 
dénotations, mais dont le caractère antiphrastique se comprend par le cotexte, qui multiplie les allusions à l'inscription des uns et des autres dans une communauté culturelle. L'énonciation ironique constitue un effet de connivence au même titre que les allusions culturelles. La catégorisation linguistique de l'ironie, insiste sur le fait que le locuteur instaure grâce à elle une connivence avec ses récepteurs (Charaudeau 2006: 32-37 $)^{12}$. Ainsi, "[l]'ironie exige la connivence du récepteur, qui devrait, pour la recevoir et surtout la goûter, avoir des valeurs et une culture commune avec l'ironiste " (Géraud 1999: 5). Si l'humour est une des composantes de l'énonciation des magazines masculins et du magazine féministe de notre corpus et l'ironie une figure de style qui n'a pas en elle-même de classe sociale, l'énonciation ironique n'est dans ces magazines pas aisément détachable des allusions culturelles et de la construction d'un profil valorisant de consommateur associé aux classes supérieures; on peut déceler dans son usage une stratégie pour construire la " connivence aristocratique », que Kierkegaard attribue à l'énonciation ironique (Kierkegaard 1975 [1841]). Si Violaine Géraud signale qu' [e]lle ne s'adresse jamais qu'à un cercle de "happy few" " (Géraud 1999:5), on peut envisager, en se plaçant dans le cadre de la linguistique pragmatique (Herschberg Pierrot 1993 : 153), qu'elle crée ce cercle de « happy few " plus qu'elle ne s'adresse à un cercle préconstruit; il s'agit de placer le lecteur dans une posture inclusive et valorisante. Bethan Benwell note ainsi que l'ironie est extrêmement présente dans le discours de la presse masculine en ce qu'elle permet de proposer une identité plurielle, riche et labile (Benwell 2004: 7) ${ }^{13}$, susceptible de correspondre à la plupart des lecteurs, tout en créant une connivence entre le magazine et son lectorat. $G Q$, dont le slogan est « masculin, beau et intelligent » (mars), titre ainsi l'éditorial consacré à l'acteur Robert Downey Jr, «Le pouvoir de l'ironie » (août), évoquant le fait que l'acteur « installe [une] espèce de second degré permanent [...] entre lui, son personnage, Hollywood et nous ». Ducrot note par ailleurs que l'ironie est créatrice à la fois de son contexte d'énonciation et de la figure d'un énonciateur qui se constitue lui-même à travers son propos (Ducrot 1984 : 216-221) ${ }^{14}$. La description du «Mec», lecteur programmatique de Lui, est ainsi paradoxale : «celui qui draguait lourdement, qui buvait trop, qui conduisait vite, qui tombait amoureux en parlant fort de politique et en faisant des moulinets avec ses petits bras musclés. Certains l'appelaient "macho", d'autres disent "néo-beauf", mais le surnom qui lui va le mieux est "connard d'hétérosexuel" " (septembre). Si l'ironie ne manifeste pas en elle-même un ancrage sociale élitaire, elle crée une " posture énonciative » relevant "d'une forme d'aisance» (Géraud 1999: 5) qui permet au locuteur de se définir lui-même en usant d'une insulte.

31 L'ironie se manifeste également par des figures de style décalées, présentes largement dans la presse masculine, mais également dans la presse féministe. Si GQ recourt à l'euphémisme («Ces dernières années n’ont pas été empreintes d'une sérénité totale s'agissant des relations hommes/femmes »; janvier), Causette manie de son côté une figure symétrique, l'hyperbole ${ }^{15}$ : "le mot "féminisme" [...] est, pour la majorité, synonyme de "femme poilue et revancharde qui picore des testicules comme vous et moi on gobe des olives en terrasse" " (mars). On retrouve dans cette description une des critiques traditionnelle faites au féminisme selon Christine Bard: «Les féministes sont violentes et antimecs » (Bard 2012 : 113), avec une forte exagération. La dimension absurde de cette figure est perceptible car elle fait suite à une comparaison : « En fait, je crois que laisser le Front national s'emparer du féminisme, c'est un peu comme confier un planning familial à Mokhtar Belmokhtar ». L'analogie mise en place dans ce cas associe ironiquement, afin de montrer leur incompatibilité, deux mouvements 
collectifs (le féminisme et le parti politique du Front National), face à une réalisation concrète du féminisme (le planning familial) et un chantre contemporain de l'antiféminisme : le djihadiste Mokhtar Belmokhtar. Des figures de pensée comme la litote, l'hyperbole ou l'ironie "témoignent toujours d'une distance à valeur appréciative par rapport au narré» (Fromilhague \& Sancier-Chateau 1999: 83). Le même magazine montre également une distance à soi-même dans une signature qui reprend les critiques traditionnellement associées aux féministes : "Causette (qui, si elle avait connu la crèche, aurait peut-être été moins revêche)»(Causette, mai). Le fait que la locutrice Causette rédige son éditorial à la première personne fait de son discours un discours incarné, ce qui lui permet d'ironiser sur elle-même. Fréderic Beigbeder fait de même dans l'éditorial de Lui. La distance à soi-même se trouve également dans la légende décalée et souvent peu valorisante évoquant l'équipe du magazine $G Q$, qui accompagne les photos anciennes et démodées illustrant l'éditorial. De même, Causette n'hésite pas à paraphraser la starlette de téléréalité Nabila (dont la citation est parodiée, alors qu'elle est citée in extenso dans le Elle du 27 mars) : " "Non mais, allô, quoi ! T'es une femme et t'es pas féministe ?! C'est comme si j'te dis t'es sanspapiers et tu votes Marine !" " (Causette, mars) pour appuyer son engagement militant. Sur le plan de l'énonciation, la presse féministe est plus proche de la presse masculine que de la presse féminine. La dimension ironique de l'énonciation des magazines masculins et du magazine féministe dresse les contours de la construction discursive du genre.

L'ironie dans son double sens d'antiphrase et de dépréciation de soi fallacieuse, qui s'inscrit dans un héritage social et établit une connivence entre le magazine et son lectorat - en l'occurrence, féminin -, construit en effet discursivement le genre, en ce qu'elle est l'opposé de ce que Lakoff avait identifié comme le comportement linguistique des femmes, qui utilisaient des «formes [reflétant] certains traits de caractère du locuteur - plus ou moins imposés par la société -, à savoir que le locuteur est indécis et qu'il n'est pas sûr de lui » (Lakoff 2004 [1975] : 286). Pascal Singy décrit ce comportement comme une "insécurité linguistique» (Singy 1998: 12 souligné par l'auteur), tout en remarquant lui aussi que ce comportement linguistique était imposé par la hiérarchie sociale ${ }^{16}$. La construction discursive du genre par l'ironie est présente dans des énoncés issus de médias masculins ou d'énoncés féminins se décrivant explicitement comme féministes. On la trouve systématiquement dans Causette, mais également, à une occasion, dans un éditorial de Elle écrit par Alix Girod de l'Ain sur la démission du Pape Benoît XVI. Or, dans cet éditorial, l'auteure se proclame explicitement féministe. La locutrice se met ainsi en scène de manière peu valorisante en caricaturant son identité chrétienne :

(7) « Le Pape démissionne, c'est un sujet pour toi ! » Après ce coup de fil de sa rédaction en chef, l'éditorialiste prend sa tête entre ses mains, perplexe. Dans le fond, c'est sa faute : tant d'années à faire sa catho-pride dans les couloirs, ces papiers qu'elle saupoudre de références bibliques, ces prières collectives qu'elle propose en conférence de rédaction afin que tout le monde s'aime les uns les autres, même les soirs de bouclage...

On y trouve également des références culturelles implicites, où l'on distingue le Tartuffe de Molière («Un pape qui démissionne, [...] c'est comme si, je sais pas moi, Karl Lagerfeld quittait Chanel (pour être chrétienne, on n'en est pas moins journaliste à $E L L E$ ) ou George Clooney prenait vingt-cinq kilos (pour être chrétienne, on n'en est pas 
moins femme) »), finissant par l'affirmation explicite de convictions féministes : «la solution, ce serait bien sûr d'élire une femme (pour être catholique, on n'en est pas moins féministe) ». Si l'on observe bien dans cet extrait la convergence d'une posture énonciative ironique et la proclamation d'un engagement féministe, on trouve toutefois des traces d'insécurité linguistique - dans les références aux injonctions de la rédaction en chef, ou dans l'emploi de l'expression «je ne sais pas moi »- qui renvoient aux ambiguïtés du magazine vis-à-vis de l'engagement féministe. Ainsi, si l'on cherche à identifier un "discours de genre" dans les magazines de notre corpus, on peut le chercher moins du côté d'une dichotomie homme-femme que du côté d'une polarisation entre, d'une part, une "insécurité linguistique», et, de l'autre, une « sécurité linguistique » faite d'aisance, de connivence, de découverte et de liberté, à la fois héritée et à conquérir - et matérialisée par une énonciation ironique.

Le rôle de l'énonciation ironique dans la construction discursive du genre est perceptible dans une autre figure d'antiphrase, celle du retournement carnavalesque des expériences liées au genre. Or, ce retournement n'est pas seulement humoristique. Ses présupposés implicites sont au contraire très révélateurs de la manière dont le titre définit les rôles de genre. En effet, ce retournement constitue soit un échange des rôles genrés (où l'inégalité est renversée mais reproduite), soit une assimilation des rôles, dans une volonté d'égalisation. Ainsi, si le positionnement énonciatif des éditorialistes est comparable dans des médias comme Lui, GQ ou Causette, l'implicite de l'énonciation ironique dresse une construction du genre qui demeure différenciée. On trouve deux retournements carnavalesques dans notre corpus. Frédéric Beigbeder présente dans Lui son quotidien possible après la supposée « victoire des femmes » :

(8) Les femmes bossent dans des bureaux, conduisent de grosses bagnoles, se soûlent entre potes dans des bars. [...]. Elles ont voulu échanger notre vie contre la leur : voilà qui est fait. [...] L'échange me convient parfaitement. Je vais être très heureux de faire la cuisine, de torcher les gosses, de remplir le lave-vaisselle, de faire les courses au supermarché, si vous me promettez que je n'aurai plus jamais à travailler. [...] Ma femme ira au boulot avec sa voiture dans les embouteillages, elle engueulera son secrétaire, lui mettra des mains aux fesses, et puis elle rentrera le soir, les enfants seront déjà au lit, je lui aurai préparé une petite blanquette de veau. (Lui, septembre).

L'inversion des rôles de genre se fait ici sur le plan des places sociales, et «l'échange ", pour reprendre le terme de Frédéric Beigbeder, est une interversion : ce qui a été gagné par les femmes a été perdu par les hommes. L'interversion des rôles de genre reprend ainsi un "leitmotiv du discours antiféministe" présent depuis le début du $\mathrm{xx}^{\mathrm{e}}$ siècle : celui de "l'homme devenu "ménagère" " (Maugue 1999:78-80). La construction du genre présente au cœur de l'énonciation ironique de Lui est ainsi implicitement liée à un antiféminisme traditionnel. Causette met en place de son côté un retournement fondé sur une assimilation de la représentation du corps des hommes et des femmes :

(9) Imaginez un peu la tête de mon brun ténébreux si [...] la radio du bar lâchait sans prévenir : "Gland malodorant? Fini les scandales conjugaux, avec Puriglan !" [...] Et si on revendiquait l'égalité par le bas? [...] Que les agences de com se mettent vite à identifier tout ce que les hommes cherchent à nous cacher et le balancent enfin à la télé et sur les bus! Éjaculation précoce? Ah ah! Démangeaisons intimes? Ah ah ah! [...] Mais, mon pauvre chouchou, c'est parce que je t'aime que je veux rire de tes petits secrets. Et un peu pour que tu saches ce que ça fait ! (Causette, janvier) 
L'éditorial de Lui évoque ce retournement en termes d' "échange ", celui de Causette se place du point de vue de la convergence des expériences. L'horizon à atteindre n'est pas une inversion, mais une égalité des situations. Si l'énonciation ironique se retrouve dans les magazines masculins et féministes, on perçoit toutefois dans l'implicite de cette énonciation une différence entre le discours masculin traditionnel et le discours féministe. Ce dernier construit en effet une connivence avec son lectorat en s'appuyant sur la généralisation des problématiques partagées par les femmes, et sur une possible assimilation des expériences masculines et féminines. La construction discursive du genre dans cette perspective féministe se veut donc inclusive - et l'ironie en est une composante essentielle. La construction d'un "discours de genre" ironique s'opposerait à la dichotomie, issue des rapports de genre traditionnels et hétéronormés, entre "discours universaliste » non marqué d'une part, et «insécurité linguistique » de l'autre. Le "discours de genre » féministe s'appuie donc à la fois sur les caractéristiques du discours féministe traditionnel, et sur celles du discours «masculin». Il s'agit d'un discours où l'expérience de la locutrice est centrale, généralisable à toutes les femmes, mais également applicable aux hommes. L'énonciation ironique, par son caractère antiphrastique et humoristique, est dès lors le moyen discursif d'unifier toutes ces caractéristiques dans un « discours de genre ».

\section{Conclusion}

Pour conclure, observer le « discours de genre » dans la presse magazine masculine et féminine en 2013 fait remarquer dans un premier temps que les thématiques énoncées s'appuient sur une dichotomie traditionnelle entre «dedans» et «dehors» pour finalement converger vers l'expression de rapports de genre en pleine évolution - que les éditorialistes s'en réjouissent ou le déplorent. Toutefois, on a pu observer une différence entre le discours énoncé par des hommes et celui énoncé par des femmes dans l'ancrage à la fois temporel et culturel des énoncés : alors que les éditoriaux des magazines féminins décrivent la féminité comme l'objet d'une lutte contemporaine à l'échelle mondiale, ceux des magazines masculins inscrivent leur masculinité programmatique dans un héritage culturel riche et partagé, mais également fondé sur une distance ironique à soi-même.

Dans cette dimension énonciative ironique, on observe les principales lignes d'évolution dans la manière de "dire le genre " : cette énonciation ironique et décalée est l'apanage à la fois des éditoriaux des magazines masculins, et de ceux qui se présentent comme explicitement féministes. Le discours féministe se fonde donc conjointement sur l'inclusion des différentes expériences, et son énonciation ironique permet d'unifier le discours à partir de l'incarnation de la figure de la locutrice. Identifier les caractéristiques du "discours de genre» permet ainsi non seulement d'appréhender la dimension genrée du discours médiatique, à la fois comme artefact et comme technologie de genre - qu'il s'agisse d'un discours explicitement genré ou non, puisqu'on en trouve des traces dans le discours de la presse générale, qui se présente comme universel et neutre -, mais également de comprendre plus précisément les mécanismes à l'œuvre dans le discours médiatique féministe dit de la troisième vague. 


\section{BIBLIOGRAPHIE}

AEBISCHER, Verena. 1985. Les femmes et le langage. Représentations d'une différence. Paris : PUF.

BARD, Christine. 2012. Le Féminisme au-delà des idées reçues. Paris : Le Cavalier Bleu. BARDELOT, Estelle. 2001. La « nouvelle presse masculine » Réseaux 105 : 161-189.

BENWELL, Bethan. 2003. « Ambiguous masculinities: heroism and anti-heroism in the men's lifestyle magazine » The Sociological Review $51: 151-168$.

BENWELL, Bethan. 2004. «Ironic Discourse: Evasive Masculinity in British Men's Lifestyle Magazines » Men and masculinities $7: 3-21$.

BOURDIEU, Pierre. 1979. La distinction. Critique du jugement social. Paris : Minuit.

BUTLER, Judith. 2005 [1990]. Trouble dans le genre. (C.Krauss, trad.) Paris : La Découverte.

CHARAUDEAU, Patrick. 2006. « Des catégories pour l'humour? » Questions de communication 10 : 19-42.

CHARON, Jean-Marie. 2001. « La presse magazine. Un média à part entière ? » Réseaux 105 : 53-78.

CONNELL, Raewyn. 2005. Masculinities. Berkeley, Los Angeles : University of California Press.

COULOMB-GULLY, Marlène \& RENNES, Juliette. 2010. « Genre, politique et analyse du discours » Mots. Les langages du politique, $94: 175-182$.

DARRAS, Éric. 2004. «Les genres de la presse féminine. Éléments pour une sociologie politique de la presse féminine » in Presse écrite : objets délaissés, Legavre Jean-Baptiste (éd.). Paris :

L'Harmattan, 271-288.

DE LAURETIS, Teresa. 2007. Théorie queer et cultures populaires : de Foucault à Cronenberg. (M.H. Bourcier, trad.) Paris : La Dispute.

DEBRAS, Sylvie. 1999. «La moitié du lectorat » in Dites-le avec des femmes. Le sexisme ordinaire dans les médias. Paris : CFD, 107-140.

DEBRAS, Sylvie. 2003. « Lectrices oubliées au quotidien » Réseaux 120 : 175-204.

DUBOIS, Jean. 1969. « Énoncé et énonciation » Langages 4-13 : 100-110.

DUCROT, Oswald. 1984. Le dire et le dit. Paris : Minuit.

DUMARSAIS, César Chesneau. 1988 [1730]. Des tropes ou des différents sens. Paris : Flammarion. DUPUIS-DÉRI, François. 2009. « Le "masculinisme" : une histoire politique du mot (en anglais et en français) » Recherches féministes $22: 97-123$.

DUPUIS-DÉRI, François. 2012. « Le discours de la "crise de la masculinité" comme refus de l'égalité entre les sexes : histoire d'une rhétorique antiféministe » Cahiers du Genre 52 : 119-143. MORIN, Edgar. 1962. L'esprit du temps. Paris : Grasset.

EDWARDS, Derek \& POTTER, Jonathan. 1992. Discursive psychology. London, Newbury Park, New Delhi : Sage Publications Ltd. 
EGGINS, Suzanne \& IEDEMA, Rick. 1997. « Difference without diversity: semantic orientation and ideology in competing women's magazines » in Gender and discourse, WODAK, Ruth (éd.). London : SAGE Publications Ltd, 165-196.

FERNANDEZ, Manuel \& VIVERO GARCIA, María Dolorès. 2006. « L'humour dans la chronique de presse quotidienne » Questions de communication $10: 81-101$.

FROMILHAGUE, Catherine \& SANCIER-CHÂTEAU, Anne. 1999. Analyses stylistiques. Paris : Dunod. GEERS, Alexie. 2016. Le sourire et le tablier. La construction médiatique du féminin dans MarieClaire de 1937 à nos jours. Thèse de Doctorat, Paris : École des Hautes Études en Sciences Sociales. GÉRAUD, Violaine. 1999. «L'ironie au siècle des Lumières » L'Information Grammaticale, 83-1 : 3-8.

GROSSE, Ernst-Ulrich \& SEIBOLD, Ernst. 1996. « Typologie des genres journalistiques » in Panorama de la presse parisienne, GROSSE, Ernst-Ulrich \& SEIBOLD, Ernst (éd.). Berlin : Peter Lang, 32-55.

GUÉRIN, Serge. 1999. «L'évolution du magazine Notre Temps : de l'ajustement à l'anticipation du marché » Communication et langages $121: 4-14$.

HÉRITIER, Françoise. 1996. Masculin, Féminin. La pensée de la différence. Paris : Odile Jacob.

HERMAN, Thierry \& JUFER, Nicole. 2001. « L'éditorial, “vitrine idéologique du journal” ? » Semen [En ligne], 13, consulté le 18 mai 2017. URL : http://semen.revues.org/2610

HERSCHBERG PIERROT, Anne. 1993. Stylistique de la prose. Paris : Belin.

JANKÉLÉVITCH, Vladimir. 1964. L'ironie. Paris : Flammarion.

JODELET, Denise. 2003. «1. Représentations sociales: un domaine en expansion » in Les représentations sociales, JODELET, Denise (ed.) Paris : PUF.

KIERKEGAARD, Søren. 1975 [1841]. Le concept d'ironie. CEuvres complètes 2. Paris : Editions de l'Orante.

LABOV, William. 1998. "Vers une réévaluation de l'insécurité linguistique des femmes » in Les femmes et la langue. L'insécurité linguistique en question, SINGY, Pascal (éd.). Lausanne, Paris : Delachaux \& Niestlé.

LAHIRE, Bernard. 2004. La culture des individus. Dissonances culturelles et distinction de soi. Paris : La Découverte.

LAKOFF, Robin Tolmach. 2004 [1975]. Language and woman's place: Text and commentaries. Oxford : Oxford University Press.

LALANDE, André. 2010 [1926]. Vocabulaire technique et critique de la philosophie. Paris : PUF.

MACÉ, Éric. 2001. «Qu'est-ce qu'une sociologie de la télévision ? 2 » Réseaux 105 : 199-242.

McILVENNY, Paul. 2002. «Introduction. Researching Talk, Gender and Sexuality » in Talking gender and sexuality, McILVENNY, Paul (éd.). Amsterdam/Philadelphia : John Benjamins Publishing, 1-48.

MAUGUE, Annelise. 1999. «Littérature antiféministe et angoisse masculine » in Un siècle d'antiféminisme, BARD, Christine (éd.). Paris : Fayard, 69-83.

NEVEU, Erik. 1993. «Pages “Politique” » Mots 37 : 6-28.

OLIVESI, Aurélie. 2012. Implicitement sexiste ? Genre, politique et discours journalistique. Toulouse : Presses Universitaires du Mirail, 155-189 
POTTER, Jonathan \& WETHERELL, Margaret. 1987. Discourse and social psychology: Beyond attitudes and behaviour. London : Sage Publications Ltd.

RAHMAN KHAN, Shamus. 2012. « The sociology of elites » Annual Review of Sociology 38 : 361-377.

RIEFFEL, Rémy. 1985. L'élite des journalistes. Paris : PUF.

RIUTORT, Philippe. 1996. « Grandir l'événement. L'art et la manière de l'éditorialiste » Réseaux $76: 61-81$.

SCOTT, Joan W. 1998 [1996]. La citoyenne paradoxale. Les féministes françaises et les droits de l'homme. (M. Bourdé \& C. Pratt, trad.) Paris : Albin Michel.

SINGY, Pascal. 1998. « Sociolinguistique, gender studies : l'insécurité linguistique en question » in Les femmes et la langue. L'insécurité linguistique en question, SINGY, Pascal (éd.). Lausanne, Paris : Delachaux et Niestlé, 9-22.

SPEER, Suzanne \& POTTER, Jontathan. 2002. « From performatives to practices Judith Butler, discursive psychology and the management of heterosexist talk » in Talking gender and sexuality, McILVENNY, Paul (éd.). Amsterdam/Philadelphia : John Benjamins Publishing, 151-180.

VIDAL, Catherine \& BENOIT-BROWAEYS, Dorothée. 2005. Cerveau, sexe \& pouvoir. Paris : Belin.

WAGNER, Anne-Catherine. 2007. Les classes sociales dans la mondialisation. Paris : La Découverte.

WODAK, Ruth (éd.). 1997. Gender and discourse. London : Sage Publications Ltd.

YOUNG, Isabel. 2016. " "If a woman pulls a knife on you, just pull out two slices of bread": an exploration of online communities of representation and the rise of 'lad culture' ", communication au séminaire Genre et Controverses en Ligne, 29 janvier 2016, Université Claude Bernard Lyon 1.

\section{NOTES}

1. Voir Potter \& Wetherell 1987, Edwards \& Potter 1992, et Speer \& Potter 2002.

2. "One of the shortcomings of Butler's work has been its limited engagement with actual texts and the detailed analysis of processes of discursive production so crucial to her hypothesis " (Speer \& Potter 2002, mentionné par Benwell 2003 : 152) Par ailleurs, l'ouvrage dirigé par Ruth Wodak sur les rapports entre genre et langage (Wodak 1997) consacre la plupart de ses chapitres au discours interpersonnel, un seul étant dédié au discours des magazines féminins (Eggins \& Iedema 1997).

3. Nous avons nous-même pu observer dans quelle mesure le discours des éditoriaux de la presse nationale d'information générale en 2007, explicitement neutre et universel, mais en pratique écrit quasi-exclusivement par des hommes (à l'exception de celui de La Croix), présentait une neutralisation imparfaite dans sa représentation des principaux candidats à l'élection présidentielle, qui en venait à présenter la candidature féminine, celle de Ségolène Royal, comme un écart par rapport à une norme implicite (Olivesi 2012).

4. Pour une approche plus documentée concernant le genre de l'éditorial, cf. Grosse \& Seibold 1996 : 49 ; Herman \& Jufer 2001 ; Neveu 1993 et Riutort 1996.

5. GQ n 64 , «Ni potes ni soumis. Les nouveaux hommes attaquent ", mai 2013 ; Causette $n^{\circ} 33$, "Égalité des sexes. Libérez l'homme nouveau! ", mars 2013 ; Books n4, « La fin du mâle », juin 2013.

6. Loi du 8 juillet 2013 pour la refondation de l'École. 
7. Causeur $n^{\circ} 7$, « Contre les lois anti-prostitution, pour la liberté : le manifeste des 343 "salauds" » illustré de l'image reprenant la main stylisée de SOS Racisme " Touche pas à ma pute », novembre 2013.

8. http://www.acpm.fr/Chiffres/Diffusion/La-Presse-Payante/Presse-Magazine

9. Pour un historique du terme « masculinisme » en anglais et en français, cf. Dupuis-Déri (2009).

10. Voir également Rahman Khan (2012).

11. The Six Million Dollar Man : série télévisée américaine de science-fiction, créée par Kenneth Johnson (1974-1978).

12. Voir également Fernandez \& Vivero Garcia (2006). Pour l'usage de l'ironie par les éditorialistes, appréhendée comme un « signe de "hauteur de vue" ", cf. Riutort (1996: 66).

13. Voir également Bardelot (2001 : 179).

14. Le pouvoir créatif de l'ironie est souligné par Jankélévitch, qui ajoute de son côté que l'ironie dans son sens romantique est créatrice de liberté heuristique: «liberté du sujet surplombant l'objet [ou] liberté magique et poétique transfigurant le monde [...]. L'ironie est le pouvoir de jouer [...], de jongler avec les contenus soit pour les nier, soit pour les recréer » (Jankélévitch $1964: 17)$.

15. «Lorsque [...] les termes ordinaires nous paraissent trop faibles pour exprimer ce que nous voulons dire, nous nous servons de mots qui, à les prendre à la lettre, vont au-delà de la vérité, et représentent le plus ou le moins pour faire entendre quelque excès en grand ou petit » (Dumarsais 1988 [1730] : 131-132).

16. L'analyse des «liens profonds et réguliers exist[a]nt entre hiérarchie sociale et structure linguistique » (Labov 1998 : 479) est reprise par Pascal Singy, qui s'attache lui aussi à dépasser la critique qui y voit une différence pensée « en termes essentialistes » (risquant de reproduire la dichotomie homme-femme traditionnelle), pour en souligner la dimension socialement construite (Singy 1998 : 12 ; voir également Aebischer 1985 : 35).

\section{RÉSUMÉS}

L'enjeu de cet article est d'observer la dimension proprement discursive de la construction du genre dans les médias. En analysant le discours des éditoriaux de magazines masculins et féminins français publiés en 2013, nous souhaitons montrer que la construction discursive du genre se trouve non seulement dans une différence de thématiques, mais également sur le plan de l'énonciation - et que le point central de cette construction est l'usage de l'ironie, à l'égard de soi-même ou de son propre discours. Après avoir observé que la divergence des thématiques entre magazines féminins et masculins renfermait une convergence de perspectives s'accordant sur une profonde évolution des rôles de genre, tout en opposant une féminité de lutte à une masculinité héritée, il s'agit de montrer à quel point la posture énonciative (ironique ou non) de l'éditorialiste se révèle structurante dans la construction d'un « discours de genre ».

In this paper, I aim at exploring how gender is built through media discourse. I have thus analysed the discourse used in editorial pieces published in French women's and men's lifestyle magazines in 2013. I show that gender, in this kind of media discourse, is not only performed through thematic choices, but is also built within discourse through enunciation itself. Therefore, even if editorialists provide similar perspectives on the evolution of gendered roles, they also offer different definitions of femininity (as the result of a fight) and masculinity (as the result of an heritage). But the most fundamental difference lies in the use of irony by editorial 
writers - toward themselves, or toward their own discourses. My conclusion is that irony contributes, in a most essential way, to the building of a gendered discourse in the media.

\section{INDEX}

Mots-clés : genre, analyse du discours, presse magazine, presse féminine, presse masculine, performativité, énonciation, ironie

Thèmes : Recherches

Keywords : gender, discourse analysis, magazines, women's magazines, men's lifestyle magazines, performativity, enunciation, irony

\section{AUTEUR}

\section{AURÉLIE OLIVESI}

Université Claude Bernard Lyon 1 - ELICO (EA 4147)

Aurélie Olivesi est maîtresse de conférences en sciences de l'information et de la communication à l'Université Claude Bernard Lyon 1 - ELICO (EA 4147). Son travail porte principalement sur la représentation médiatique du genre, et sur la parole profane dans le discours médiatique, essentiellement dans le domaine politique. Elle a publié une monographie consacrée à l'élection présidentielle de 2007 (Implicitement sexiste? Genre, politique et discours journalistique, PUM, 2012), et codirigé deux publications collectives : L'assignation de genre dans les médias, PUR, 2013, et Gender Testing in Sport. Ethics, cases and controversies, Routledge, 2016, ainsi que le numéro 6 de la revue Politiques de Communication: Des vraies gens aux followers, 2016. 